\title{
Design, Synthesis and Antifungal Activity of New Schiff Bases Bearing 2-Aminothiophene Derivatives Obtained by Molecular Simplification
}

\author{
Isadora S. Luna, ${ }^{a}$ Wendell W. Neves, ${ }^{b}$ Reginaldo G. de Lima-Neto, ${ }^{c}$ \\ Amanda P. B. Albuquerque, ${ }^{d}$ Maíra G. R. Pitta, ${ }^{d}$ Moacyr J. B. M. Rêgo, ${ }^{d}$ \\ Rejane P. Neves, ${ }^{e}$ Marcus T. Scotti ${ }^{\oplus *, a}$ and Francisco J. B. Mendonça-Junior ${ }^{\oplus a, f}$ \\ aPrograma de Pós-Graduação em Produtos Naturais e Sintéticos Bioativos, \\ Departamento de Ciências Farmacêuticas, Universidade Federal da Paraíba, 58051-900 João Pessoa-PB, Brazil \\ ${ }^{b}$ Programa de Pós-Graduação em Inovação Terapêutica, Universidade Federal de Pernambuco, \\ 50670-901 Recife-PE, Brazil \\ 'Departamento de Medicina Tropical, Universidade Federal de Pernambuco, 50670-901 Recife-PE, Brazil \\ ${ }^{d}$ Departamento de Bioquímica, Universidade Federal de Pernambuco, 50670-420 Recife-PE, Brazil \\ ${ }^{e}$ Departamento de Micologia Médica, Universidade Federal de Pernambuco, 50760-420 Recife-PE, Brazil \\ ${ }^{f}$ Departamento de Ciências Biológicas, Universidade Estadual da Paraíba, 58071-160 Recife-PE, Brazil
}

\begin{abstract}
Seventeen Schiff bases bearing 2-aminothiophene derivatives were designed and synthesized using molecular simplification. The resulting compounds (4a-4q) were evaluated for their in vitro antifungal activity against dermatophytes. Prediction of their druglikeness and pharmacokinetic properties, establishment of their structure-activity relationships (SAR), and cytotoxic evaluation of the most active compounds were performed. Using an eco-friendly procedure, microwave assisted synthesis resulted in compounds in good yields (35-85\%). Compounds $\mathbf{4 a - 4 q}$ presented good druglikeness and pharmacokinetic profiles and no cytotoxicity for any cell line tested up to $100 \mu \mathrm{M}$. The compounds presenting the best antifungal profiles were $\mathbf{4 e}, \mathbf{4 f}, \mathbf{4 g}, \mathbf{4 k}, \mathbf{4 l}, \mathbf{4 m}, \mathbf{4 0}$ and $4 \mathbf{p}$ with more than one minimum inhibitory concentration (MIC) value occurring between 16-64 $\mu \mathrm{g} \mathrm{mL}^{-1}$, thus, in some cases better than the reference drug (fluconazole). SAR testing indicated that the presence of halogens and nitro substituents increases antifungal activity. Taken together, the results demonstrate that 2-aminothiophene derivatives are promising lead compounds for the development of antifungal drugs.
\end{abstract}

Keywords: 2-aminothiophene, ADMET properties, antifungal agents, drug design, Schiff base

\section{Introduction}

During the past few decades, the incidence of fungal infection has substantially increased worldwide, and dermatophytoses, being one of the most common mycoses in humans, represent approximately $25 \%$ of this increase. ${ }^{1,2}$ Dermatophytes are filamentous fungi that cause dermatophytosis or tinea. They belong to the oldest groups of microorganisms and are recognized as agents of human disease, infecting keratinized tissues such as skin, hair and nails. ${ }^{3,4}$ The emergence of resistant dermatophytes in recent years has become a

*e-mail: mtscotti@gmail.com matter of worldwide concern, provoking interest in the development of new drugs with antifungal potential. ${ }^{5,6}$

Despite the existence of various classes of antifungal agents such as azoles, polyenes, allylamines, antimetabolites, and echinocandins, the search for new agents against pathogenic fungi is of great importance and urgency, since the currently available antifungal drugs present severe side effects and toxicity. The most common side effects associated with the use of antifungals are serious: nephrotoxicity is observed with amphotericin B, the most common polyene, ${ }^{7}$ hepatotoxicity is associated with all azoles, ${ }^{8}$ hepatotoxicity and bone marrow depression are caused by flucytosine, ${ }^{9}$ heart disease is associated with echinocandin, ${ }^{10}$ and gastrointestinal and 
hepatobiliary disorders are associated with the use of terbinafine. ${ }^{11}$

As if this was not enough, antifungal resistance to all of these drugs is common and often reported. This is directly reflected in increased morbidity and mortality. ${ }^{12,13}$ There is a real need for new antifungal drugs.

Drug design and development is a complex and multidisciplinary process that aims to obtain new chemical entities with both the desired pharmacological effect and clinical safety (toxicity thus being absent or negligible). To achieve such characteristics, a crucial step is the choice of a pharmacophore group (or groups) that will lend to the drug candidate an appropriate pharmacodynamic and pharmacokinetic (absorption, distribution, metabolism, excretion and toxicity (ADMET)) profile.

Due to their great chemical versatility and valuable pharmacological properties, Schiff bases and thiophene derivatives are widely known. ${ }^{14-18}$ Their roles in the field of antifungal therapy stand out. Commercial antifungals with applications in dermatology, such as sertaconazole, oxiconazole, ${ }^{19}$ and imibenconazole ${ }^{20}$ (Figure 1) present these pharmacophoric groups. Other examples of antifungal candidates presenting Schiff bases or thiophene scaffolds in their chemical structures are presented in Figure 1. These include: (i) Schiff bases containing a 2,4-dichloro-5-fluorophenyl group (I), being equipotent to fluconazole against Aspergillus flavus, A. fumigatus, Penicillium marneffei and Trichophyton mentagrophytes strains; ${ }^{21}$ (ii) Schiff bases bearing salicylaldehyde (II) with anti-Candida albicans activity comparable to fluconazole, ${ }^{22}$ (iii) imine containing a kakuol moiety (III) against phytopathogenic fungi, especially against Alternaria alternata; ${ }^{23}$ (iv) Schiff bases containing piperonyl (IV) capable of inhibiting the growth of Epidermophyton floccosum and Trichophyton rubrum $;^{24}$ and (v) Schiff bases of 4-(4-aminophenyl)-morpholine (V) with antifungal properties against Aspergillus niger and C. albicans. ${ }^{25}$

In recent years, our research group has investigated the potential of Schiff bases bearing cycloalka[b]thiophene derivatives for inhibiting the growth of various fungal species, especially Cryptococcus neoformans. ${ }^{26-28}$ Various compounds, such as 2-(4-nitrobenzylidene)-amino4,5,6,7-tetrahydro-4H-benzo[b]thiophene-3-carbonitrile (named 6CN10) (VI) (Figure 1) are promising. However, the low water solubility of these compounds is a limiting factor, especially when conducting in vivo studies. ${ }^{27}$ To overcome these limits, since 2014, efforts have been made to prepare adequate formulations that contain the most promising Schiff bases; those bearing cycloalka $[b]$ thiophene derivatives. Inclusion complexes with 2-hydroxypropyl- $\beta$-cyclodextrin (HP- $\beta$-CD), ${ }^{29}$ microemulsions, ${ }^{30}$ and nanoparticles (nanospheres/ nanocapsules $)^{31}$ have been prepared with a view to increase water solubility and antifungal activity. In view of these considerations, and continuing our<smiles>O=C(O)OCc1ccc(Cl)cc1Cl</smiles><smiles>O=C(O)c1ccc(Cl)cc1C(CCn1ccnc1)OCc1csc2c(Cl)cccc12</smiles><smiles>COc1ccc(/C=N/n2c(-c3cc(F)c(Cl)cc3Cl)n[nH]c2=S)cc1</smiles><smiles>O=S(=O)([O-])c1ccc(/N=C/c2cc(Cl)cc(Cl)c2O)cc1</smiles><smiles>CCOc1cc2c(cc1/N=C/c1ccccc1Cl)OCO2</smiles><smiles>Clc1ccc(/N=C/c2ccc3c(c2)OCO3)cc1</smiles><smiles>Clc1ccc(CSC(=Nc2ccc(Cl)cc2Cl)n2cncn2)cc1</smiles><smiles>COc1cc(C=Nc2ccc(N3CCOCC3)cc2)ccc1O</smiles><smiles>N#Cc1c(/N=C/c2ccc([N+](=O)[O-])cc2)sc2c1CCCC2</smiles>

Figure 1. Antifungal agents under development or marketed containing Schiff bases and thiophene derivatives. 


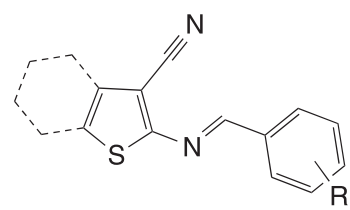

PROTOTYPE

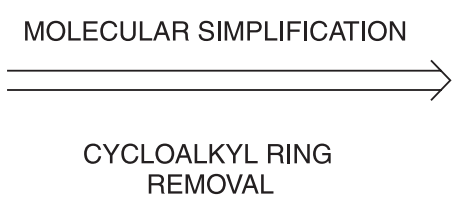

REMOVAL

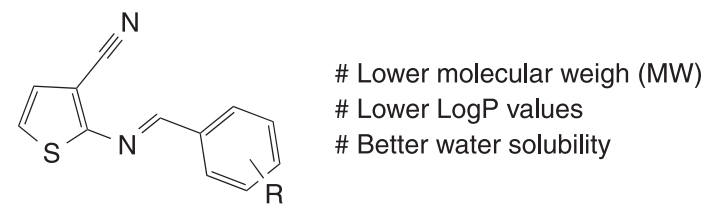

Better pharmacokinetic profile? Increase antifungal activity?

Figure 2. Rationale for the synthesis of new Schiff bases bearing 2-aminothiophene derivatives with improved antifungal activity.

program to develop compounds with potent antifungal activity, we have (through a strategy of molecular simplification $)^{32-34}$ designed a new series of Schiff bases bearing 2-aminothiophene derivatives obtained from 1,4-dithiane-2,5-diol. This makes it possible to obtain unsubstituted Gewald adducts C-4 and C-5 (Figure 2). ${ }^{35,36}$ The new compounds present lower partition coefficient (octanol-water) $(\log \mathrm{P})$ values when compared with their cycloalka[b]thiophene analogues, ${ }^{26,28}$ improving both water solubility and their pharmacokinetic profiles; and resulting in a potential increase in antifungal activity.

The aim of this study was to evaluate the in vitro antifungal activity against dermatophytes of new Schiff bases bearing 2-aminothiophene derivatives, synthesized using a molecular simplification strategy. Prediction of their druglikeness and pharmacokinetic ADMET properties, establishment of their structure-activity relationships, and cytotoxic evaluation of the most active compounds were also performed.

\section{Experimental}

\section{Chemistry}

All reagents and solvents were purchased from SigmaAldrich (St. Louis, USA), Fluka (Buchs, Switzerland), Neon (Suzano, Brazil), Vetec (Duque de Caxias, Brazil) and used without further purification. Melting points were measured using a model MA-381 melting point (Marconi, São Paulo, Brazil), apparatus and are uncorrected. Synthesis under microwave irradiation was carried out on a DiscoverSystem (CEM, Laguna Hills, USA). ${ }^{1} \mathrm{H}$ nuclear magnetic resonance (NMR), ${ }^{13} \mathrm{C}$ NMR, two-dimensional NMR (heteronuclear single quantum coherence spectroscopy (HSQC) and heteronuclear multiple bond correlation (HMBC)) spectra were recorded in dimethyl sulfoxide $\left(\right.$ DMSO- $d_{6}$ ) on a Varian Unity Plus $400 \mathrm{MHz}$ spectrometer (Bruker, Billerica, USA). Chemical shifts $(\delta)$ are given in parts per million (ppm). Multiplicities are reported as singlet (s), doublet (d), doublet of doublets (dd), triplet (t), quartet (q) and multiplet (m), and the observed coupling constants $(J)$ provided in hertz $(\mathrm{Hz})$. Peaks are assigned where possible. Infrared spectra were acquired on a VERTEX 70 series FTIR spectrometer (Bruker, Billerica, USA) using $\mathrm{KBr}$ discs, and the wavenumbers related to the transmittance minima $\left(v_{\max }\right)$ in $\mathrm{cm}^{-1}$ are reported. Exact mass measurements of the molecular ions were obtained on a Bruker Daltonics the micrOTOF-Q IITM ESI-QqTOF mass spectrometer (Bruker, Billerica, USA). The chromatographic analysis had reaction progress monitored by thin layer chromatography using Merck (Darmstadt, Germany) TLC Silica gel $60 \mathrm{~F}_{254}$ aluminium sheets and detection by short and long wavelength ultraviolet light (254 and $365 \mathrm{~nm}$ ).

\section{General}

General procedure for the synthesis of compounds $4 a-4 q$

Method A: in a round bottom flask were added 1 equiv of 1,4-dithiane-2,5-diol (1) and 2 equiv of malononitrile (2). Methanol (MeOH) was added to dissolve the mixture, and then triethylamine ( 0.6 equiv) was added dropwise. The reaction proceeded at $60{ }^{\circ} \mathrm{C}$ with stirring for $4 \mathrm{~h}$. After $4 \mathrm{~h}$, substituted aromatic aldehydes ( 2 equiv) and acetic acid ( 0.7 equiv) were added in the reaction medium, and the reaction proceeded at room temperature overnight. After completion of the reaction, the final products $(\mathbf{4} \mathbf{a}-\mathbf{4 q} \mathbf{q})$ were filtered and washed successively with cold water and $\mathrm{MeOH}$ to obtain chromatographically pure solid residues.

Method B: in a microwave tube were added 1 equiv of 1,4-dithiane-2,5-diol (1), 2 equiv of malononitrile (2), sodium bicarbonate (1.3 equiv) and $2 \mathrm{~mL}$ of ethylene glycol. The reaction mixture was irradiated $(150 \mathrm{~W})$ at $80{ }^{\circ} \mathrm{C}$ for $8 \mathrm{~min}$ (ramp time: $5 \mathrm{~min}$ ) in the microwave apparatus. After cooling, the microwave tube was opened, and were added substituted aromatic aldehydes (2 equiv), acetic acid (1.5 equiv) and $0.5 \mathrm{~mL}$ of ethylene glycol. Then, the reactional mixture was subjected under microwave irradiation $(150 \mathrm{~W})$ at $50{ }^{\circ} \mathrm{C}$ for $2 \mathrm{~min}(\mathrm{ramp}$ time: 2 min $30 \mathrm{~s}$ ). After completion of the reaction, the final products $(\mathbf{4 a - 4 q})$ were filtered and washed successively with cold $\mathrm{MeOH} /$ water (1:1) to obtain chromatographically pure solid residues. 
2-(4-Methoxybenzylidene)aminothiophene-3-carbonitrile (4a)

Green crystals; yield $86 \%(\operatorname{method} A), 85 \%(\operatorname{method} B)$; mp 133.4-135.2 ${ }^{\circ} \mathrm{C}$; IR (KBr) v / $\mathrm{cm}^{-1} 3101,2227,1593$; ${ }^{1} \mathrm{H}$ NMR (400 MHz, DMSO- $\left.d_{6}\right) \delta 3.61\left(\mathrm{~s}, 3 \mathrm{H}, \mathrm{OCH}_{3}\right), 6.87$ (d, $2 \mathrm{H}, J 8.8 \mathrm{~Hz}, \mathrm{Ph}-\mathrm{H}), 7.11$ (d, 1H, J $5.6 \mathrm{~Hz}, \mathrm{H}-4), 7.25$ (d, $1 \mathrm{H}, J 6.0 \mathrm{~Hz}, \mathrm{H}-5), 7.71$ (d, 2H, J 8.8 Hz, Ph-H), 8.43 $(\mathrm{s}, 1 \mathrm{H}, \mathrm{C} \underline{\mathrm{H}}=\mathrm{N}) ;{ }^{13} \mathrm{C}$ NMR $\left(100 \mathrm{MHz}, \mathrm{DMSO}-d_{6}\right) \delta 56.0$, 106.1, 115.1, 115.3, 123.1, 124.5, 127.9, 129.4, 132.0, 162.2; HRMS (ESI) $m / z$, calcd. for $\mathrm{C}_{13} \mathrm{H}_{10} \mathrm{~N}_{2} \mathrm{OS}[\mathrm{H}]^{+}$: 243.0595, found: 243.0593 .

2-(4-Chlorobenzylidene)aminothiophene-3-carbonitrile (4b)

Green crystals; yield 66\% (method A), 52\% (method B); mp 104.3-105.9 ${ }^{\circ} \mathrm{C}$; IR (KBr) v / $\mathrm{cm}^{-1} 3111,2229,1590$; ${ }^{1} \mathrm{HNMR}\left(400 \mathrm{MHz}, \mathrm{DMSO}-d_{6}\right) \delta 7.39(\mathrm{~d}, 1 \mathrm{H}, J 5.6 \mathrm{~Hz}, \mathrm{H}-4)$, 7.58 (d, 1H, J 5.6 Hz, H-5), 7.61 (d, 2H, J $1.4 \mathrm{~Hz}, \mathrm{Ph}-\mathrm{H})$, 7.99 (d, 2H, J 1.0 Hz, Ph-H), 8.77 (s, 1H, C $\underline{H}=\mathrm{N}) ;{ }^{13} \mathrm{C} \mathrm{NMR}$ $\left(100 \mathrm{MHz}, \mathrm{DMSO}-d_{6}\right) \delta 99.4,105.6,114.4,123.8,127.5$, 129.1, 130.8, 133.4, 137.3, 161.0; HRMS (ESI) $\mathrm{m} / z$, calcd. for $\mathrm{C}_{12} \mathrm{H}_{7} \mathrm{ClN}_{2} \mathrm{~S}[\mathrm{H}]^{+}:$247.0095, found: 247.0097.

2-(4-Fluorobenzylidene)aminothiophene-3-carbonitrile (4c)

Green crystals; yield 55\% (method A), 66\% (method B); mp 109.3-110.1 ${ }^{\circ} \mathrm{C}$; IR (KBr) v / $\mathrm{cm}^{-1} 3128,2228,1580$; ${ }^{1} \mathrm{H}$ NMR (400 MHz, DMSO- $\left.d_{6}\right) \delta 7.43(\mathrm{~d}, 1 \mathrm{H}, J 6.0 \mathrm{~Hz}$, H-4), 7.65 (d, 1H, J 5.6 Hz, H-5), 7.85 (t, 1H, J 8.0 Hz, Ph-H), 8.39-8.42 (m, 2H, Ph-H), 8.77-8.78 (m, 1H, Ph-H), $8.95(\mathrm{~s}, 1 \mathrm{H}, \mathrm{C} \underline{\mathrm{H}}=\mathrm{N}) ;{ }^{13} \mathrm{C}$ NMR $\left(100 \mathrm{MHz}, \mathrm{DMSO}-d_{6}\right)$ $\delta$ 107.0, 114.9, 124.0, 125.2, 127.1, 128.3, 131.2, 135.3, 136.7, 161.0; HRMS (ESI) $\mathrm{m} / 2$, calcd. for $\mathrm{C}_{12} \mathrm{H}_{7} \mathrm{FN}_{2} \mathrm{~S}[\mathrm{H}]^{+}$: 231.0370, found: 231.0393 .

2-(2,4-Dichlorobenzylidene)aminothiophene-3-carbonitrile (4d)

Yellow crystals; yield 49\% (method A), 35\% (method B); mp 152.6-153 ${ }^{\circ} \mathrm{C}$; IR (KBr) v / $\mathrm{cm}^{-1} 3121,2232,1582$; ${ }^{1} \mathrm{H}$ NMR (400 MHz, DMSO- $\left.d_{6}\right) \delta 7.43(\mathrm{~d}, 1 \mathrm{H}, J 5.6 \mathrm{~Hz}$, $\mathrm{H}-4), 7.61$ (dd, 1H, J 8.4, $2.0 \mathrm{~Hz}, \mathrm{Ph}-\mathrm{H}), 7.65$ (d, 1H, $J 5.6 \mathrm{~Hz}, \mathrm{H}-5), 7.80(\mathrm{~d}, 1 \mathrm{H}, J 2.0 \mathrm{~Hz}, \mathrm{Ph}-\mathrm{H}), 8.15(\mathrm{~d}, 1 \mathrm{H}$, $J 8.8 \mathrm{~Hz}, \mathrm{Ph}-\mathrm{H}), 8.83(\mathrm{~s}, 1 \mathrm{H}, \mathrm{CH}=\mathrm{N}) ;{ }^{13} \mathrm{C}$ NMR $(100 \mathrm{MHz}$, DMSO- $\left.d_{6}\right) \delta 106.9,114.8,125.2,126.8,128.4,128.8,130.3$, 130.4, 132.8, 136.8, 138.4, 156.8; HRMS (ESI) $\mathrm{m} / z$, calcd. for $\mathrm{C}_{12} \mathrm{H}_{6} \mathrm{Cl}_{2} \mathrm{~N}_{2} \mathrm{~S}[\mathrm{H}]^{+}: 280.9700$, found: 280.9708 .

\section{2-(2,6-Dichlorobenzylidene)aminothiophene-3-carbonitrile} $(4 e)$

Yellow crystals; yield $56 \%(\operatorname{method} \mathrm{A}), 83 \%(\operatorname{method} \mathrm{B})$; mp 150.1-151.5 ${ }^{\circ} \mathrm{C}$; IR (KBr) v / $\mathrm{cm}^{-1} 3107,2225,1578$; ${ }^{1} \mathrm{H}$ NMR (400 MHz, DMSO- $\left.d_{6}\right) \delta 7.46(\mathrm{~d}, 1 \mathrm{H}, J 5.6 \mathrm{~Hz}$, H-4), 7.55 (dd, 1H, J 8.8, 7.2 Hz, Ph-H), 7.63 (d, 2H,
$J 8.0 \mathrm{~Hz}, \mathrm{Ph}-\mathrm{H}), 7.69$ (d, 1H, J 5.6 Hz, H-5), 8.90 (s, 1H, $\mathrm{C} \underline{\mathrm{H}}=\mathrm{N}) ;{ }^{13} \mathrm{C}$ NMR $\left(100 \mathrm{MHz}, \mathrm{DMSO}-d_{6}\right) \delta 106.6,114.2$, 125.1, 128.1, 129.6, 130.2, 132.8, 134.7, 157.9, 161.5; HRMS (ESI) $m / z$, calcd. for $\mathrm{C}_{12} \mathrm{H}_{6} \mathrm{Cl}_{2} \mathrm{~N}_{2} \mathrm{~S}[\mathrm{H}]^{+}: 280.9707$, found: 280.9708 .

2-(2-Nitrobenzylidene)aminothiophene-3-carbonitrile (4f)

Green crystals; yield 44\% (method A), 52\% (method B); mp 179.8-80.3 ${ }^{\circ} \mathrm{C}$; IR (KBr) v / cm ${ }^{-1} 3100,2233,1509$; ${ }^{1} \mathrm{H}$ NMR $\left(400 \mathrm{MHz}, \mathrm{DMSO}-d_{6}\right) \delta 7.46(\mathrm{~d}, 1 \mathrm{H}, J 5.6 \mathrm{~Hz}$, H-4), 7.66 (d, 1H, J 5.6 Hz, H-5), $7.83(\mathrm{t}, 1 \mathrm{H}, J 7.2 \mathrm{~Hz}$, Ph-H), 7.92 (t, 1H, J 7.6 Hz, Ph-H), 8.16 (d, 1H, J $8.0 \mathrm{~Hz}$, Ph-H), 8.21 (d, 1H, J 7.6 Hz, Ph-H), 9.04 (s, 1H, C $\underline{H}=\mathrm{N})$; ${ }^{13} \mathrm{C}$ NMR (100 MHz, DMSO- $\left.d_{6}\right) \delta 108.8,114.7,117.2$, 125.3, 125.4, 128.5, 130.0, 131.5, 133.4, 134.5, 159.1, 164.5; HRMS (ESI) $m / z$, calcd. for $\mathrm{C}_{12} \mathrm{H}_{7} \mathrm{~N}_{3} \mathrm{O}_{2} \mathrm{~S}[\mathrm{H}]^{+}$: 258.0329, found: 258.0338 .

2-(3-Nitrobenzylidene)aminothiophene-3-carbonitrile (4g)

Green crystals; yield 64\% (method A), 78\% (method B); mp 196.3-197.6 ${ }^{\circ} \mathrm{C}$; IR (KBr) v / $\mathrm{cm}^{-1} 3096,2232,1522$; ${ }^{1} \mathrm{H}$ NMR $\left(400 \mathrm{MHz}, \mathrm{DMSO}-d_{6}\right) \delta 7.19(\mathrm{~d}, 1 \mathrm{H}, J 5.6 \mathrm{~Hz}$, $\mathrm{H}-4), 7.41$ (d, 1H, J 5.6 Hz, H-5), 7.60 (t, 1H, J $8.0 \mathrm{~Hz}$, Ph-H), 8.14-8.18 (m, 2H, Ph-H), 8.53 (t, 1H, J $2.0 \mathrm{~Hz}$, $\mathrm{Ph}-\mathrm{H}), 8.71(\mathrm{~s}, 1 \mathrm{H}, \mathrm{CH}=\mathrm{N}) ;{ }^{13} \mathrm{C}$ NMR $(100 \mathrm{MHz}$, DMSO- $\left.d_{6}\right) \delta 107.0,114.9,124.0,125.2,127.1,127.2$, 128.3, 131.2, 135.3, 136.7, 160.9, 161.0; HRMS (ESI) $\mathrm{m} / \mathrm{z}$, calcd. for $\mathrm{C}_{12} \mathrm{H}_{7} \mathrm{~N}_{3} \mathrm{O}_{2} \mathrm{~S}[\mathrm{H}]^{+}:$258.0333, found: 258.0338.

2-(2-Hydroxybenzylidene)aminothiophene-3-carbonitrile (4h)

Green crystals; yield 45\% (method A), 79\% (method B); mp 131.5-133.4 ${ }^{\circ} \mathrm{C}$; IR (KBr) v / $\mathrm{cm}^{-1} 3115,2228,1604$; ${ }^{1} \mathrm{H}$ NMR (400 MHz, DMSO- $\left.d_{6}\right) \delta 6.98-7.02(\mathrm{~m}, 2 \mathrm{H}, \mathrm{Ph}-\mathrm{H})$, 7.40 (d, 1H, J 5.6 Hz, H-4), 7.47 (dt, 1H, J 7.2, 1.6 Hz, Ph-H), $7.58(\mathrm{~d}, 1 \mathrm{H}, J 5.6 \mathrm{~Hz}, \mathrm{H}-5), 7.82(\mathrm{dd}, 1 \mathrm{H}, J 8.0,1.6 \mathrm{~Hz}$, $\mathrm{Ph}-\mathrm{H}), 8.97$ (s, 1H, CH=N), 11.49 (s, 1H, OH); ${ }^{13} \mathrm{C}$ NMR $\left(100 \mathrm{MHz}, \mathrm{DMSO}-d_{6}\right) \delta 104.7,114.4,116.8,119.4,119.7$, 123.5, 127.3, 131.0, 134.8, 159.9, 161.7, 162.2; HRMS (ESI) $\mathrm{m} / z$, calcd. for $\mathrm{C}_{12} \mathrm{H}_{8} \mathrm{~N}_{2} \mathrm{OS}[\mathrm{H}]^{+}: 229.0427$, found: 229.0454 .

2-(4-Methylbenzylidene)aminothiophene-3-carbonitrile (4i) Green crystals; yield 59\% (method A), 27\% (method B); mp 116.5-117.1 ${ }^{\circ} \mathrm{C}$; IR (KBr) v / $\mathrm{cm}^{-1} 3109,2222,1599$; ${ }^{1} \mathrm{H}$ NMR (400 MHz, DMSO-d $\left.d_{6}\right) \delta 2.39$ (s, 3H, $\mathrm{CH}_{3}$ ), 7.37 (d, 2H, J 8.0 Hz, Ph-H), 7.38 (d, 1H, J 5.6 Hz, H-4), 7.54 (d, 1H, J 5.6 Hz, H-5), 7.90 (d, 2H, J 8.0 Hz, Ph-H), 8.72 $(\mathrm{s}, 1 \mathrm{H}, \mathrm{C} \underline{\mathrm{H}}=\mathrm{N}) ;{ }^{13} \mathrm{C}$ NMR $\left(100 \mathrm{MHz}, \mathrm{DMSO}-d_{6}\right) \delta 21.3$, 104.8, 114.7, 123.2, 127.5, 129.4, 129.7, 132.0, 143.3, 162.3, 163.4; HRMS (ESI) $\mathrm{m} / \mathrm{z}$, calcd. for $\mathrm{C}_{13} \mathrm{H}_{10} \mathrm{~N}_{2} \mathrm{OS}$ $[\mathrm{H}]^{+}:$227.0626, found: 227.0644 . 
2-\{(4-(Dimethylamino)benzylideneamino\}thiophene3-carbonitrile (4j)

Orange crystals; yield $31 \%$ (method A), 55\% (method B); mp 143.5-144.3 ${ }^{\circ} \mathrm{C}$; IR (KBr) $v / \mathrm{cm}^{-1} 3094$, 2225, 1579; ${ }^{1} \mathrm{H}$ NMR (400 MHz, DMSO- $d_{6}$ ) $\delta 3.05$ (s, $\left.6 \mathrm{H}, \mathrm{CH}_{3}\right), 6.81(\mathrm{~d}, 2 \mathrm{H}, J 8.8 \mathrm{~Hz}, \mathrm{Ph}-\mathrm{H}), 7.28(\mathrm{~d}, 1 \mathrm{H}$, $J 5.6 \mathrm{~Hz}, \mathrm{H}-4), 7.37$ (d, $1 \mathrm{H}, J 6.0 \mathrm{~Hz}, \mathrm{H}-5), 7.81$ (d, 2H, $J 8.8 \mathrm{~Hz}, \mathrm{Ph}-\mathrm{H}), 8.49$ (s, $1 \mathrm{H}, \mathrm{CH}=\mathrm{N}) ;{ }^{13} \mathrm{C}$ NMR $(100 \mathrm{MHz}$, DMSO- $\left.d_{6}\right) \delta 39.6,102.2,111.5,115.2,120.9,121.7,127.1$, 131.5, 153.3, 161.5, 165.3; HRMS (ESI) $\mathrm{m} / \mathrm{z}$, calcd. for $\mathrm{C}_{14} \mathrm{H}_{13} \mathrm{~N}_{3} \mathrm{~S}[\mathrm{H}]^{+}: 256.0914$, found: 256.0909 .

\section{2-(4-Nitrobenzylidene)aminothiophene-3-carbonitrile (4k)}

Brown crystals; yield $60 \%$ (method A), $90 \%$ (method B); mp 153.8-155.4 ${ }^{\circ} \mathrm{C}$; IR (KBr) v / $\mathrm{cm}^{-1} 3093,2230,1720$; ${ }^{1} \mathrm{H}$ NMR (400 MHz, DMSO- $\left.d_{6}\right) \delta 7.47(\mathrm{~d}, 1 \mathrm{H}, J 5.6 \mathrm{~Hz}$, H-4), 7.70 (d, 1H, J 5.6 Hz, H-5), 8.25 (d, 2H, J $8.8 \mathrm{~Hz}$, $\mathrm{Ph}-\mathrm{H}), 8.41$ (d, 2H, J 8.4 Hz, Ph-H), 8.95 (s, 1H, CH=N); ${ }^{13} \mathrm{C}$ NMR $\left(100 \mathrm{MHz}\right.$, DMSO- $\left.d_{6}\right) \delta 107.9,115.3,124.7$, 125.1, 126.1, 128.9, 131.2, 131.5, 141.1, 150.3, 161.3, 162.8; HRMS (ESI) $\mathrm{m} / z$, calcd. for $\mathrm{C}_{12} \mathrm{H}_{7} \mathrm{~N}_{3} \mathrm{O}_{2} \mathrm{~S}[\mathrm{H}]^{+}$: 258.0347, found: 258.0338 .

\section{2-(3,4,5-Trimethoxybenzylidene)aminothiophene-} 3-carbonitrile (4I)

Yellow crystals; yield 39\% (method A), 57\% (method B); mp 160.6-161.7 ${ }^{\circ} \mathrm{C}$; IR (KBr) $v / \mathrm{cm}^{-1} 3091,2222,1570$; ${ }^{1} \mathrm{H} \mathrm{NMR}\left(400 \mathrm{MHz}, \mathrm{DMSO}-d_{6}\right.$ ) $\delta 3.77$ (s, $3 \mathrm{H}, \mathrm{OCH}_{3}$ ), 3.86 (s, $\left.6 \mathrm{H}, \mathrm{OCH}_{3}\right), 7.36(\mathrm{~s}, 2 \mathrm{H}, \mathrm{Ph}-\mathrm{H}), 7.39(\mathrm{~d}, 1 \mathrm{H}, J 5.6 \mathrm{~Hz}$, $\mathrm{H}-4), 7.55$ (d, 1H, J 5.6 Hz, H-5), 8.69 (s, 1H, CH=N); ${ }^{13} \mathrm{C}$ NMR $\left(100 \mathrm{MHz}\right.$, DMSO- $\left.d_{6}\right) \delta 55.9,60.2,104.6,106.7$, 114.7, 123.2, 127.6, 129.9, 141.6, 153.1, 162.2, 163.3; HRMS (ESI) $\mathrm{m} / z$, calcd. for $\mathrm{C}_{15} \mathrm{H}_{14} \mathrm{~N}_{2} \mathrm{O}_{3} \mathrm{~S}[\mathrm{H}]^{+}: 303.0795$, found: 303.0804 .

\section{2-(Benzylidene)aminothiophene-3-carbonitrile (4m)}

Green crystals; yield 49\% (method A), 63\% (method B); mp 113.3-114.1 ${ }^{\circ} \mathrm{C}$; IR (KBr) v / cm ${ }^{-1} 3096,2224,1571$; ${ }^{1} \mathrm{H}$ NMR $\left(400 \mathrm{MHz}\right.$, DMSO- $\left.d_{6}\right) \delta 7.40(\mathrm{~d}, 1 \mathrm{H}, J 6.0 \mathrm{~Hz}$, $\mathrm{H}-4)$, 7.55-7.63 (m, 4H, Ph-H + H-5), $8.01(\mathrm{~d}, 2 \mathrm{H}$, $J 6.8 \mathrm{~Hz}, \mathrm{Ph}-\mathrm{H}), 8.78(\mathrm{~s}, 1 \mathrm{H}, \mathrm{CH}=\mathrm{N}) ;{ }^{13} \mathrm{C}$ NMR $(100 \mathrm{MHz}$, DMSO- $\left.d_{6}\right) \delta 105.7,115.1,124.1,128.1,129.5,129.9$, 133.3, 135.1, 163.0, 163.6; HRMS (ESI) $\mathrm{m} / \mathrm{z}$, calcd. for $\mathrm{C}_{14} \mathrm{H}_{14} \mathrm{~N}_{2} \mathrm{~S}[\mathrm{H}]^{+}:$213.0472, found: 213.0487.

\section{2-(2,3-Dichlorobenzylidene)aminothiophene-3-carbonitrile} (4n)

Yellow crystals; yield $56 \%$ (method A), $46 \%$ (method B); mp 175.5-176.4 ${ }^{\circ} \mathrm{C}$; IR (KBr) v / $\mathrm{cm}^{-1} 3092$, 2236, $1552 ;{ }^{1} \mathrm{H}$ NMR $\left(400 \mathrm{MHz}\right.$, DMSO- $\left.d_{6}\right) \delta 7.46(\mathrm{~d}, 1 \mathrm{H}$, $J 6.0 \mathrm{~Hz}, \mathrm{H}-4), 7.57$ (t, 1H, J 8.0 Hz, Ph-H), 7.68 (d, 1H,
$J 5.6 \mathrm{~Hz}, \mathrm{H}-5), 7.88$ (d, 1H, J $8.0 \mathrm{~Hz}, \mathrm{Ph}-\mathrm{H}), 8.15$ (d, 1H, $J 8.0 \mathrm{~Hz}, \mathrm{Ph}-\mathrm{H}), 8.94(\mathrm{~s}, 1 \mathrm{H}, \mathrm{CH}=\mathrm{N}) ;{ }^{13} \mathrm{C}$ NMR $(100 \mathrm{MHz}$, DMSO- $\left.d_{6}\right) \delta 107.2,114.8,125.5,127.8,128.5,129.2$, 132.2, 133.8, 134.2, 134.5, 157.8, 162.3; HRMS (ESI) $\mathrm{m} / z$, calcd. for $\mathrm{C}_{12} \mathrm{H}_{6} \mathrm{Cl}_{2} \mathrm{~N}_{2} \mathrm{~S}[\mathrm{H}]^{+}:$280.9696, found: 280.9708 .

2-(4-Bromobenzylidene)aminothiophene-3-carbonitrile (40)

Green crystals; yield $46 \%$ (method A), 63\% (method B); mp 95.8-98.4 ${ }^{\circ} \mathrm{C}$; IR (KBr) v / $\mathrm{cm}^{-1} 3094,2230,1584$; ${ }^{1} \mathrm{H}$ NMR $\left(400 \mathrm{MHz}\right.$, DMSO- $\left.d_{6}\right) \delta 7.41(\mathrm{~d}, 1 \mathrm{H}, J 5.6 \mathrm{~Hz}$, H-4), 7.60 (d, 1H, J 6.0 Hz, H-5), 7.77 (d, 2H, J 8.8 Hz, $\mathrm{Ph}-\mathrm{H}), 7.94$ (d, 2H, J $8.8 \mathrm{~Hz}, \mathrm{Ph}-\mathrm{H}), 8.78$ (s, 1H, CH=N); ${ }^{13} \mathrm{C}$ NMR $\left(100 \mathrm{MHz}\right.$, DMSO- $\left.d_{6}\right) \delta 105.7,114.5,124.0$, 126.5, 127.7, 131.0, 132.1, 133.8, 161.3, 162.7; HRMS (ESI) $\mathrm{m} / z$, calcd. for $\mathrm{C}_{12} \mathrm{H}_{7} \mathrm{BrN}_{2} \mathrm{~S}[\mathrm{H}]^{+}: 290.9590$, found: 290.9592 .

2-\{(4-Nitro- $1 H$-indol-3-yl)methyleneamino $\}$ thiophene3-carbonitrile (4p)

Yellow crystals; yield 64\% (method A), 50\% (method B); mp 172-174. ${ }^{\circ} \mathrm{C}$; IR (KBr) v / $\mathrm{cm}^{-1} 3225,2231,1512$; ${ }^{1} \mathrm{H}$ NMR (400 MHz, DMSO- $\left.d_{6}\right) \delta 7.31(\mathrm{~d}, 1 \mathrm{H}, J 5.6 \mathrm{~Hz}$, H-4), 7.40 (d, 1H, J 6.4 Hz, H-5), 7.44 (d, 1H, J 8.0 Hz, Ph-H), 7.96 (d, 1H, J 8.4 Hz, Ph-H), 8.00 (d, 1H, J 8.0 Hz, Ph-H ), 8.58 (s, 1H, Ph-H), 9.10 (s, 1H, CH=N), 12.93 (sl, $1 \mathrm{H}, \mathrm{NH}) ;{ }^{13} \mathrm{C}$ NMR $\left(100 \mathrm{MHz}\right.$, DMSO- $\left.d_{6}\right) \delta 102.2,112.3$, 115.0, 117.1, 119.2, 119.6, 121.1, 122.1, 127.4, 135.5, 139.5, 142.1, 158.3, 165.3; HRMS (ESI) $\mathrm{m} / \mathrm{z}$, calcd. for $\mathrm{C}_{14} \mathrm{H}_{8} \mathrm{~N}_{4} \mathrm{OS}[\mathrm{H}]^{+}:$297.0427, found: 297.0447.

2-\{(5-Methoxy-1H-indol-3-yl)methyleneamino\}thiophene3-carbonitrile (4q)

Blue crystals; yield 23\% (method A), 71\% (method B); mp 278.4-281.3 ${ }^{\circ} \mathrm{C}$; IR (KBr) $v / \mathrm{cm}^{-1} 3305,2218,1578$; ${ }^{1} \mathrm{H}$ NMR $\left(400 \mathrm{MHz}, \mathrm{DMSO}-d_{6}\right) \delta 3.82\left(\mathrm{~s}, 3 \mathrm{H}, \mathrm{OCH}_{3}\right)$, 6.91 (dd, 1H, J 8.8, 2.4 Hz, Ph-H), 7.30 (d, 1H, J 6.0 Hz, H-4), 7.35 (d, 1H, J 6.0 Hz, H-5), 7.42 (d, 1H, J $8.8 \mathrm{~Hz}$, Ph-H), 8.06 (d, 1H, J 2.8 Hz, Ph-H), 8.15 (d, 1H, J 3.2 Hz, $\mathrm{Ph}-\mathrm{H}), 8.82$ (s, $1 \mathrm{H}, \mathrm{CH}=\mathrm{N}), 12.02(\mathrm{sl}, 1 \mathrm{H}, \mathrm{NH}) ;{ }^{13} \mathrm{C}$ NMR $\left(100 \mathrm{MHz}, \mathrm{DMSO}-d_{6}\right) \delta 55.1,102.3,103.9,113.1,113.4$, $114.1,115.5,120.2,125.4,126.6,132.0,136.7,155.4$, 156.4, 166.2; HRMS (ESI) $\mathrm{m} / \mathrm{z}$, calcd. for $\mathrm{C}_{15} \mathrm{H}_{11} \mathrm{~N}_{3} \mathrm{OS}$ $[\mathrm{H}]^{+}$: 282.0714, found: 282.0702 .

\section{Prediction of biological activity}

Prediction of druglikeness and pharmacokinetics (ADMET) properties

The druglikeness, pharmacokinetics properties, and toxicity profile of compounds $\mathbf{4} \mathbf{a}-\mathbf{4 q}$ were determined using an ADMET descriptors algorithm protocol of 
pkCSM. ${ }^{37}$ This software has been used as evidence to train accurate molecular predictors of important physicochemical parameters such as molecular weight (MW), topological polar surface area (TPSA), partition coefficient (octanol-water) (LogP), number of hydrogen bond acceptor (nHBA), number of hydrogen bond donors (nHBD) and rotatable bonds (ROTB). Analysis of these aspects were used to verify the druglikeness properties of 2-aminothiophenes based on the guidelines of the Lipinski's and Weber's rules..$^{38}$ Absorption properties were analyzed based on membrane permeability (indicated by colon cancer cell line (Caco-2) permeability), skin permeability and the categorical classification of 2-aminothiophene derivatives. Drug distribution was predicted according the blood-brain barrier permeability $(\log B \mathrm{~B})$ and central nervous system permeability ( $\log P S)$. Metabolism property was evaluated based on the CYP models for substrate or inhibition (CYP2D6, CYP3A4, CYP1A2, CYP2C19, CYP2C9, CYP2D6 and CYP3A4). Excretion aspect was predicted by the total clearance model and the categorical classification of 2-aminothiophene derivatives as a renal OCT2 substrate. Toxicity of compounds was obtained based on AMES toxicity, hERG I and II inhibition, oral rat acute and chronic toxicity, hepatotoxicity, skin sensitization, T. pyriformis toxicity and Minnow toxicity. After the results, all the pharmacokinetic parameters were calculated and checked for compliance with their standard ranges. ${ }^{39}$

Pharmacological/biological assays

\section{Antifungal activity}

\section{Preparation of samples and standards}

All compounds (4a-4q) were dissolved in dimethylsulfoxide (DMSO). Fluconazole (used as reference drug) was solubilized in sterile distilled water. Then, all solutions were filtered through a $22 \mathrm{~mm}$ diameter filters. Subsequently, all drugs solutions were diluted in Roswell Park Memorial Institute (RPMI) 1640 medium buffered with 4-morpholinepropanesulfonic acid (MOPS), to obtain $1.024 \mu \mathrm{g} \mathrm{mL}^{-1}$ solutions. Compounds $\mathbf{4 a - 4 q}$ were tested in concentrations ranged from 1 to $1.024 \mu \mathrm{g} \mathrm{mL}^{-1}$ and fluconazol in concentrations ranging from 0.125 to $64 \mu \mathrm{g} \mathrm{mL} L^{-1}$.

\section{Antifungal sensitivity}

E. floccosum, T. tonsurans, T. mentagrophytes, T. rubrum and C. parapsilosis ATCC 22019 (reference strain) were used in the test were deposited in Micoteca URM of the Mycology Department of the Federal University of Pernambuco. The tests were performed according to Clinical Laboratory Standard Institute (CLSI), standard M38-A2. ${ }^{40}$ Dermatophytes species were grown on Sabouraud dextrose agar (Merck KGaA, Darmstadt, Germany) at $24^{\circ} \mathrm{C}$ four to seven days. For the test, it was removed an inoculum culture and suspended in $5.0 \mathrm{~mL}$ of sterile saline $0.085 \%$ and placed in vortex for $15 \mathrm{~s}$. The cell density was adjusted by a spectrophotometer Genesys UV-VIS 10S (Thermo Scientific, Waltham, USA) to obtain the transmittance equivalent to a standard solution of $0.5 \mathrm{McF}$ arland wavelength of $530 \mathrm{~nm}$. The suspension for testing was obtained by making a 1/100 dilution followed by a 1/50 dilution in RPMI 1640 medium with MOPS for dermatophytes.

\section{Cytotoxicity}

\section{Cell culture}

Immortalized non-tumor cell lines from human fibroblasts (MRC-05), monkey kidney epithelial cells (VERO) and murine fibroblasts (3T3) from the Bank of Cells of Rio de Janeiro and stocked in the Tumor Cell Bank of LINAT/UFPE. VERO and MRC-05 cells were cultured in low glucose Dubelcco's modified eagle medium (DMEM) and 3T3 cells in RPMI 1640 medium both supplemented with $10 \%$ bovine fetal serum, $10 \mathrm{mM}$ 4-(2-hydroxyethyl)-1-piperazineethanesulfonic acid (HEPES) and 10,000 $\mathrm{U} \mathrm{mL}^{-1}$ penicillin/streptomycin. The medium of the MRC-05 cells was supplemented with $20 \%$ bovine fetal serum, $10 \%$ insulin and $10 \%$ fibroblast growth factor. The lineages were kept in an oven containing 5\% $\mathrm{CO}_{2}$ at $37{ }^{\circ} \mathrm{C}$ and routinely evaluated for mycoplasma contamination.

\section{Cytotoxicity in non-transformed cells}

Cells were plated $\left(1 \times 10^{4}\right.$ cells well $\left.^{-1}\right)$ and incubated in a humid atmosphere containing $5 \% \mathrm{CO}_{2}$ at $37^{\circ} \mathrm{C}$ for $24 \mathrm{~h}$. Then, the $\mathbf{4 e}, \mathbf{4 f}, \mathbf{4 g}, \mathbf{4 k}, \mathbf{4 l}, \mathbf{4 m}$ and $\mathbf{4 o}$ derivatives were added at the concentrations of $1,10,50$ and $100 \mu \mathrm{M}$ and the plates were incubated for $72 \mathrm{~h}$. Vehicle control (DMSO) was added at the same concentration present in the final solutions. Then, $20 \mu \mathrm{L}$ of the 3-(4,5-dimethylthiazol-2-yl)2,5-diphenyltetrazolium bromide (MTT) solution were added to each well and after $3 \mathrm{~h}$ of incubation and $130 \mu \mathrm{L}$ of $20 \%$ of dodecyl sulfate sodium (SDS) were added for the dissolution of the formazan crystals, keeping the reaction sheltered from light for $24 \mathrm{~h}$. After this period, the optical density was read in a spectrophotometer in the microplate reader EL808 (Biotek $^{\circledR}$, Winooski, USA) at $570 \mathrm{~nm}$ absorbance. The mean optical density of the test conditions was compared to the vehicle mean $0.5 \%$ for the determination of cell viability. 


\section{Statistical analysis}

The analysis of mean, standard deviation and viability was performed in Excel $^{\circledR}$ software. ${ }^{41}$ In all the tests three independent experiments were performed and each condition was evaluated in technical replicates.

\section{Results and Discussion}

\section{Chemistry}

The Schiff bases bearing 2-aminothiophene derivatives (4a-4q) were obtained through a molecular simplification strategy being synthesized in a two-step reaction in two procedures, as shown in Scheme 1. 2-Aminothiophene3-carbonitrile (3) was synthesized using the fourth version of Gewald-type reaction. ${ }^{35}$ Reacting commercial 1,4-dithiane-2,5-diol (1), with malononitrile (2) in methanol and triethylamine and magnetic stirring (method A), or in ethylene glycol and sodium bicarbonate under microwave irradiation (method B), we obtained compounds $\mathbf{4 a - 4 q . ~ V a r i o u s ~ a r o m a t i c ~ a l d e h y d e s ~ w e r e ~ a d d e d ~}$ to each reaction media, along with a few drops of acetic acid, to yield the target compounds in yields ranging from $23-86 \%(\operatorname{method} \mathrm{A})$ and $35-85 \%(\operatorname{method} \mathrm{B})$ (Table 1$).$ The compound structures were confirmed using NMR $\left({ }^{1} \mathrm{H} \mathrm{NMR},{ }^{13} \mathrm{C}\right.$ NMR, HSQC and heteronuclear multiple bond correlation (HMBC)), infrared (IR), and highresolution mass spectrum (HRMS) analyses (available in the Supplementary Information (SI) section).

The synthetic methodologies used to obtain the target compounds, conventional (method A) and microwave irradiation (method B) proved to be both viable and efficient. Hesse et al. ${ }^{42}$ obtained 2-aminothiophene3-carbonitrile (compound 3), using both microwave and conventional methods in respective yields of 60 and $58 \%$. In our procedures, compound $\mathbf{3}$ was obtained without
Table 1. Chemical structures and yields of the Schiff bases bearing 2-aminothiophene derivatives obtained by conventional (method A) and microwave irradiation (method B) methods

\begin{tabular}{|c|c|c|c|c|}
\hline \multirow{2}{*}{ Compound } & \multirow{2}{*}{$\mathrm{R}$} & \multirow{2}{*}{$\mathrm{R}^{\prime}$} & \multicolumn{2}{|c|}{ Yield / \% } \\
\hline & & & Method A & Method B \\
\hline $4 a$ & $4-\mathrm{OCH}_{3}$ & - & 86 & 85 \\
\hline $4 b$ & $4-\mathrm{Cl}$ & - & 65.6 & 52 \\
\hline $4 c$ & $4-\mathrm{F}$ & - & 54.9 & 66 \\
\hline $4 d$ & $2,4-\mathrm{Cl}$ & - & 49 & 35 \\
\hline $4 e$ & $2,6-\mathrm{Cl}$ & - & 56 & 83 \\
\hline $4 f$ & $2-\mathrm{NO}_{2}$ & - & 44 & 52 \\
\hline $4 g$ & $3-\mathrm{NO}_{2}$ & - & 65 & 78 \\
\hline $4 h$ & $4-\mathrm{OH}$ & - & 45 & 79 \\
\hline $4 i$ & $4-\mathrm{CH}_{3}$ & - & 59 & 66 \\
\hline $4 j$ & $4-\mathrm{N}\left(\mathrm{CH}_{3}\right)_{2}$ & - & 31 & 55 \\
\hline $4 k$ & $4-\mathrm{NO}_{2}$ & - & 60 & 49 \\
\hline 41 & $3,4,5-\mathrm{OCH}_{3}$ & - & 39 & 56 \\
\hline $4 m$ & $\mathrm{H}$ & - & 49 & 44 \\
\hline $4 n$ & $2,3-\mathrm{Cl}$ & - & 56 & 64 \\
\hline 40 & $4-\mathrm{Br}$ & - & 47 & 45 \\
\hline $4 p$ & - & $4-\mathrm{NO}_{2}$ & 64 & 50 \\
\hline $4 q$ & - & $5-\mathrm{OCH}_{3}$ & 23 & 71 \\
\hline
\end{tabular}

isolation, and after reacting with aromatic aldehydes it provided several final compounds in final global yields greater than 60\% (after two-step reaction), demonstrating that our procedures were better than previously described.

The reactions conducted using method $\mathrm{B}$ were more efficient (providing better yields) in $60 \%$ of the procedures (11 of 18 reactions). The microwave assisted syntheses of Schiff bases bearing 2-aminothiophene derivatives presented specific advantages such as: shorter reaction time (10 min), energy savings, and the use of an inorganic base (sodium bicarbonate) instead of the traditional organic<smiles>CC(C)CCCCC#N</smiles><smiles>N#Cc1ccsc1N</smiles>

3

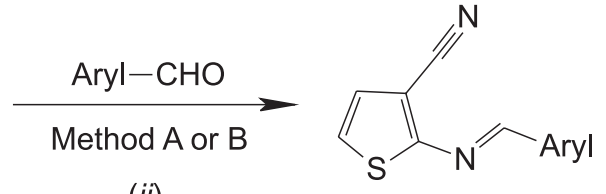

(ii)

\section{$4 a-4 q$}

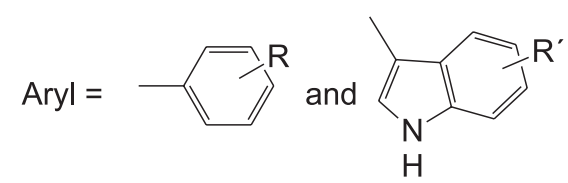

Scheme 1. Synthesis of Schiff bases bearing 2-aminothiophene derivatives (4a-4q). Method A: (i) triethylamine, methanol, $60^{\circ} \mathrm{C}, 4 \mathrm{~h}$; (ii) acetic acid, rt, overnight. Method B: (i) sodium bicarbonate, ethylene glycol, microwave, $80^{\circ} \mathrm{C}$ for $8 \mathrm{~min}$; (ii) acetic acid, microwave, $50^{\circ} \mathrm{C}, 2 \mathrm{~min}$. 
bases used in Gewald-type reactions, an eco-friendly procedure. ${ }^{43}$

\section{Druglikeness prediction}

The druglikeness of compounds (4a-4q) was determined based on Lipinski's and Veber's rules. As can be seen in Table 2, none of the compounds violated Lipinski's rule of five (presenting no more than $5 \mathrm{HBD}$; presenting no more than $10 \mathrm{HBA}$; having a MW of less than $500 \mathrm{Da}$; and presenting $\operatorname{LogP}$ of less than 5). ${ }^{38,44}$ Likewise, none of the Weber parameters were breached: presenting a PSA of less than $140 \AA$; and presenting less than 10 ROTB. ${ }^{37,45}$ Based on these data, the compounds possess a drug candidate profile with good oral bioavailability.

\section{Prediction of pharmacokinetic (ADMET) properties}

In the process of drug discovery and development, prediction of pharmacokinetic properties is a common procedure which during the initial planning phase allows identification of compounds with poor ADMET properties. This reduces the number of compounds to be tested in the early stages of research, and also the number of compounds which might fail in clinical trials. ${ }^{46-48}$ pKCSM software ${ }^{37}$ was used to predict certain ADMET parameters for compounds $\mathbf{4 a - 4 q}$ (Table S1 with all results is available in the SI section).

The absorption of orally administered drugs depends on their ability to cross the gastrointestinal tract (GIT) walls. ${ }^{49}$ Caco- 2 cells extracted from human epithelial colorectal adenocarcinoma are widely used since these cells mimic the gastrointestinal epithelium. They present a validated assay system for oral absorption studies. ${ }^{50-52}$ Compounds with a Papp (apparent permeability coefficient) value of $>0.90$ present high Caco-2 permeability, and so, the potential for GIT absorption. ${ }^{37}$ The data presented in Table S1 show that compounds $\mathbf{4 a - 4 q}$ (except those presenting nitro substituents: $\mathbf{4 f}, \mathbf{4 g}, \mathbf{4 k}$ and $\mathbf{4 p}$ ) present high Caco-2 permeability, indicating that the compounds are highly likely to be absorbed by the GIT.

Skin permeation was also predicted, and most of compounds (except indolic derivatives $\mathbf{4 p}$ and $\mathbf{4 q}$ ) demonstrated relatively low skin permeability, $\log \mathrm{Kp}>$ $-2.5 .^{37}$ This value indicates that if administrated topically, the compounds will not be well absorbed by the skin.

Distribution analysis (concerning the ability to leave circulation and enter extravascular tissues) of compounds $\mathbf{4 a - 4 q}$ was based on blood brain barrier (BBB) and central nervous system (CNS) permeability (Table S1).

BBB permeability is an important parameter since it is composed of endothelial cells which regulate (as a

Table 2. Druglikeness of compounds 4a-4q based on Lipinski (partition coefficient (LogP); hydrogen bond acceptor (HBA); hydrogen bond donor (HBD); molecular weight (MW)) and Weber (rotatable bond number (ROTB); polar surface area (PSA)) rules

\begin{tabular}{|c|c|c|c|c|c|c|}
\hline \multirow{2}{*}{ Compound } & \multicolumn{4}{|c|}{ Lipinski's } & \multicolumn{2}{|c|}{ Weber's } \\
\hline & $\log \mathrm{P}$ & HBA & HBD & $\mathrm{MW} / \mathrm{Da}$ & ROTB & PSA / A \\
\hline $4 a$ & 3.3 & 4 & 0 & 242.303 & 3 & 103.625 \\
\hline $4 b$ & 4.0 & 3 & 0 & 246.722 & 2 & 102.450 \\
\hline $4 c$ & 3.5 & 3 & 0 & 230.267 & 2 & 96.312 \\
\hline $4 d$ & 4.6 & 3 & 0 & 281.167 & 2 & 112.753 \\
\hline $4 e$ & 4.6 & 3 & 0 & 281.167 & 2 & 112.753 \\
\hline $4 f$ & 3.2 & 5 & 0 & 257.274 & 3 & 106.800 \\
\hline $4 g$ & 3.2 & 5 & 0 & 257.274 & 3 & 106.800 \\
\hline $4 h$ & 3.0 & 4 & 1 & 228.276 & 2 & 96.941 \\
\hline $4 i$ & 3.6 & 3 & 0 & 226.304 & 2 & 98.512 \\
\hline $4 j$ & 3.4 & 4 & 0 & 255.346 & 3 & 110.636 \\
\hline $4 k$ & 3.2 & 5 & 0 & 257.274 & 3 & 106.800 \\
\hline 41 & 3.3 & 3 & 0 & 212.277 & 2 & 92.147 \\
\hline $4 m$ & 4.1 & 3 & 0 & 291.173 & 2 & 106.014 \\
\hline $4 n$ & 4.0 & 3 & 0 & 246.722 & 2 & 102.450 \\
\hline 40 & 5.0 & 3 & 0 & 288.375 & 3 & 127.204 \\
\hline $4 p$ & 3.7 & 5 & 1 & 296.311 & 3 & 122.690 \\
\hline $4 q$ & 3.8 & 4 & 1 & 281.340 & 3 & 119.516 \\
\hline
\end{tabular}


protective function) the passage of compounds from the blood to the CNS. ${ }^{49}$

According to the pkCSM model, compounds with a $\log \mathrm{BB}>0.3$ can readily cross the BBB while compounds with a $\log \mathrm{BB}<-1$ present difficulty in distribution to the brain. Compounds with a $\log \mathrm{PS}>-2$ can penetrate to the CNS, while those with a $\log \mathrm{PS}<-3$ are considered unable to penetrate to the $\mathrm{CNS} .{ }^{37}$

With regard to distribution, compounds $\mathbf{4 a - 4 q}$ presented intermediate BBB permeability values, suggesting somewhat limited brain distribution, yet they were able to penetrate and likely present effects at the CNS level.

First pass metabolic characteristics depend on interaction with several microsomal enzymes known as the cytochrome P450 (CYP450) superfamily. ${ }^{49}$ These enzymes are mostly located in the liver being responsible for the majority of first-pass drug metabolism. CYP3A4 performs almost 50\% of xenobiotic metabolism in humans and is highlighted. ${ }^{53,54}$

ADMET predictions revealed that compounds $\mathbf{4 a - 4 q}$ are substrate to CYP3A4 isoforms, and non-substrate to CYP2D6 isoforms, excepting compound $\mathbf{4 q}$ for CYP3A4, and 40 for CYP2D6. The compounds were also inhibitors of CYP1A2, CYP2C19, and CYP2C9 isoforms, and noninhibitors of CYP2D6, and CYP3A4 isoforms, (except compound $\mathbf{4 o}$ for CYP2C9, and $\mathbf{4 p}$ for CYP3A4) (Table S1).

Excretion is another important pharmacokinetic parameter related to removal of an intact drug or its metabolites from the body, thus determining the amount of time the drug will remain in the organism as well as its volume of distribution. ${ }^{47,53,54}$

This parameter was analyzed based on total clearance (values were estimated, and from this, the rate of excretion can be predicted) and evaluation of the behavior of the compounds as a renal OCT2 substrate (Table S1). There is no delimited range for total clearance, the higher the value, the faster the excretion process will be. ${ }^{37}$

OCT2 is a renal uptake transporter responsible for renal drug clearance. ${ }^{55}$ The ability of a compound to bind to OCT2 is an indication of its clearance which (to achieve a steady-state in the blood plasma) is important for determining posology. ${ }^{56}$ In accordance with Table S1, compounds $\mathbf{4 a - 4 q}$ were unable to interact with or bind OCT2, which limits excretion using this pathway.

Drug toxicity is the most common impairment of drug discovery and development. ${ }^{57}$ Using pkCSM, induced toxicity was evaluated for the Schiff bases bearing 2-aminothiophenes (Table S1).

Genetic toxicity was assessed by the AMES test; screening compounds $\mathbf{4 a - 4 q}$ for mutagenic or nonmutagenic potential. Compounds that present a positive AMES test result may be mutagenic..$^{37,58}$ The results revealed seven compounds with a negative result (probably not mutagenic), while ten compounds were positive, presenting risks of mutagenicity.

Cardiotoxicity was evaluated by testing whether the compounds might be hERG I and/or hERG II inhibitors. hERG channels play an important role in cardiac repolarization. ${ }^{59-61}$ hERG current inhibition is the most likely mechanism involved in drug-induced QT (measure of delayed ventricular repolarisation) interval prolongation, and severe cardiac arrhythmias are an important reason for drug failures during preclinical study. ${ }^{37,54}$ Excepting compounds $\mathbf{4 o}$ and $\mathbf{4 q}$ (which tested positive for hERG II inhibition), compounds $\mathbf{4 a - 4 q}$ did not test positive for either hERG I or hERG II inhibition.

A principal motive for post-market removal of a medication is hepatotoxicity. ${ }^{62}$ Drug-induced liver injury can lead to acute liver failure and even death. ${ }^{63,64} \mathrm{In}$ accordance with Table S1, compounds $\mathbf{4 a - 4 q}$ were unable to disrupt normal liver function and are therefore not considered hepatotoxic.

Skin sensitization is a potential adverse effect of drugs that are applied topically. ${ }^{37}$ Table S1 reveals that the compounds $\mathbf{4 a - 4 q}$, except $\mathbf{4 m}$, presented no skin sensitization potential.

Tetrahymena pyriformis is a protozoan whose toxicity is often used as a toxic endpoint. Compounds with $\log$ IGC (population growth impairment toxicity) values of $>-0.5$ are considered toxic. ${ }^{37,47}$ All of the $\log$ IGC values were greater than -0.5 , and no compounds presented toxicity to T. pyriformis.

\section{Antifungal activity}

The in vitro antifungal activity of compounds $\mathbf{4 a - 4 q}$ together with fluconazole (reference drug) against 8 dermatophyte fungi strains including E. floccosum, T. tonsurans, T. mentagrophytes, T. rubrum and Candida parapsilosis ATCC 22019 (reference strain) was determined using the microdilution broth method according to the guidelines of the CLSI, standard M38-A2. ${ }^{40}$ The minimum inhibitory concentration (MIC) values are summarized in Table 3. For each antifungal susceptibility experiment, the inoculum controls presented clear detectable growth after the incubation period, indicating that all of the isolates were viable and that the conditions were suitable for fungal growth. The MIC of fluconazole against the reference strain was $2.0 \mu \mathrm{g} \mathrm{mL} \mathrm{L}^{-1}$, confirming the assay's reproducibility.

The results revealed that all of the compounds exhibit antifungal activity in different degrees for at least one of the analyzed dermatophytes. The compounds with the better antifungal profiles were $\mathbf{4 e}, \mathbf{4 f}, \mathbf{4 g}, \mathbf{4 k}, \mathbf{4 l}, \mathbf{4 m}, \mathbf{4 0}$ 
and $\mathbf{4 p}$, each presenting at least one MIC value ranging between 16-64 $\mu \mathrm{g} \mathrm{mL}^{-1}$. This in some cases was better than or equivalent to the reference drug fluconazole. In the case of the reference drug fluconazole, it can be seen that (with the exception of the reference strain ATCC 22019 and E. floccosum 6754) all isolates were either resistant (MIC $\geq 64 \mu \mathrm{g} \mathrm{mL}^{-1}$ ) or presented inhibited growth in a dose-dependent pattern $\left(\mathrm{MIC}=16\right.$ or $\left.32 \mu \mathrm{g} \mathrm{mL}^{-1}\right)$. $^{65,66}$

E. floccosum 6754 proved to be more resistant to the 2-aminothiophene derivatives than E. floccosum 6999, which in high concentrations (MICs $=512$ and $\left.1,024 \mu \mathrm{g} \mathrm{mL}^{-1}\right)$ was sensitive to only 6 compounds $(\mathbf{4 a}$, 4h, 4i, 4k, 4m and 4o). E. floccosum 6999 was sensitive to 14 compounds, presenting MIC values of up to $16 \mu \mathrm{g} \mathrm{mL} \mathrm{L}^{-1}$ (for $\mathbf{4 m}$ ), being equipotent to fluconazole.

For all of the Trichophyton isolates tested, it was observed that about half of the compounds inhibited the growth of the fungi. Against T. tonsurans, compound $\mathbf{4 m}$ presented MIC values of $32 \mu \mathrm{g} \mathrm{mL}{ }^{-1}$, for both isolates, a better profile than that of the reference drug. Compounds $4 \mathbf{e}$ and 40 presented MIC values of $64 \mu \mathrm{g} \mathrm{mL}^{-1}$ against T. tonsurans 700 , also superior to fluconazole. T. rubrum 6753 was the most sensitive isolate, with 5 compounds testing as equipotent to fluconazole (MIC $=64 \mu \mathrm{g} \mathrm{mL}^{-1}$, $\mathbf{4 k}, \mathbf{4 l}$ and $\mathbf{4 p}$ ) or better (MIC $=32 \mu \mathrm{g} \mathrm{mL} \mathrm{m}^{-1}, \mathbf{4 e}$ and $\left.\mathbf{4 o}\right)$.

\section{Cytotoxicity of selected compounds}

The most active compounds (4e, $4 \mathbf{f}, \mathbf{4 g}, \mathbf{4 k}, \mathbf{4 l}, \mathbf{4 m}$ and 4o) were evaluated for cytotoxicity at concentrations of 1-100 $\mu \mathrm{M}$, using the MTT assay ${ }^{67}$ with three non-tumor cell lines (VERO, MRC-05 and 3T3).

No difference in cell viability was observed for any of the compounds, at any of the concentrations evaluated, after $72 \mathrm{~h}$ of incubation. All of the compounds presented similar profiles. None of the compounds showed toxicity for any of the cell lines to the highest concentration evaluated $(100 \mu \mathrm{M})($ Figure S1 and Table S2 of cell viability for each cell line are available in the SI section).

\section{Structure-activity relationships (SAR)}

Based on the results of the antifungal activity in Table 3, the presence of nitro substituents $\left(\mathrm{NO}_{2}\right)$ attached to the arylidene moiety (as observed in compounds $\mathbf{4 f}, \mathbf{4 g}, \mathbf{4 k}$ and $\mathbf{4 p}$ ) is important for the antifungal activity. The results demonstrate that all of the tested compounds containing nitro substituents presented better activity profiles (with MIC values of less than or equal to the reference drug (MIC $\left.\geq 64 \mu \mathrm{g} \mathrm{mL}^{-1}\right)$ ). For the benzylidene derivatives presenting nitro substituents in positions 2,3 , and 4

Table 3. Antifungal activity of compounds $\mathbf{4 a - 4 q}$ and fluconazole against dermatophyte isolates

\begin{tabular}{|c|c|c|c|c|c|c|c|c|}
\hline Compound $^{\mathrm{a}}$ & $\begin{array}{l}\text { C. parapsilosis } \\
\text { ATCC }^{\mathrm{b}} 22019 / \\
\quad\left(\mu \mathrm{g} \mathrm{mL} \mathrm{mL}^{-1}\right)\end{array}$ & $\begin{array}{c}\text { E. floccosum } \\
6999 / \\
\left.(\mu \mathrm{g} \mathrm{mL})^{-1}\right)\end{array}$ & $\begin{array}{c}\text { E. floccosum } \\
6754 / \\
\left(\mu \mathrm{g} \mathrm{mL}^{-1}\right)\end{array}$ & $\begin{array}{l}\text { T. tonsurans } \\
\quad 700 / \\
\left.(\mu \mathrm{g} \mathrm{mL})^{-1}\right)\end{array}$ & $\begin{array}{l}\text { T. tonsurans } \\
2822 / \\
\left.(\mu \mathrm{g} \mathrm{mL})^{-1}\right)\end{array}$ & $\begin{array}{c}\text { T. mentagrophytes } \\
5431 / \\
\left(\mu \mathrm{g} \mathrm{mL}^{-1}\right)\end{array}$ & $\begin{array}{c}\text { T. mentagrophytes } \\
5432 / \\
\left(\mu \mathrm{g} \mathrm{mL}^{-1}\right)\end{array}$ & $\begin{array}{c}\text { T. rubrum } \\
6753 / \\
\left(\mu \mathrm{g} \mathrm{mL}^{-1}\right)\end{array}$ \\
\hline $4 a$ & 1.024 & 256 & 1.024 & 1.024 & 1.024 & 1.024 & 1.024 & 1.024 \\
\hline $4 b$ & 1.024 & 128 & $\mathrm{G}$ & G & 1.024 & 1.024 & 1.024 & 1.024 \\
\hline $4 c$ & 512 & 256 & G & 256 & G & 256 & 512 & $\mathrm{G}$ \\
\hline $4 d$ & $\mathrm{G}$ & 128 & G & G & G & G & G & G \\
\hline $4 e$ & G & G & G & 64 & 256 & G & G & 32 \\
\hline $4 f$ & 128 & 32 & G & G & G & G & $\mathrm{G}$ & G \\
\hline $4 \mathrm{~g}$ & G & 64 & G & G & G & $\mathrm{G}$ & G & 128 \\
\hline $4 h$ & 1.024 & $\mathrm{G}$ & 1.024 & 1.024 & 1.024 & $\mathrm{G}$ & 1.024 & $\mathrm{G}$ \\
\hline $4 i$ & $\mathrm{G}$ & $\mathrm{G}$ & 51 & $\mathrm{G}$ & $\mathrm{G}$ & 128 & 512 & G \\
\hline $4 j$ & 1.024 & 128 & G & 256 & 1.024 & 1.024 & 512 & 1.024 \\
\hline $4 k$ & 1.024 & 1.024 & 512 & 512 & G & G & G & 64 \\
\hline 41 & G & 256 & G & 128 & G & 256 & 128 & 64 \\
\hline $4 m$ & G & 16 & 512 & 32 & 32 & G & 256 & G \\
\hline 4n & 512 & 256 & $\mathrm{G}$ & $\mathrm{G}$ & $\mathrm{G}$ & $\mathrm{G}$ & $\mathrm{G}$ & G \\
\hline 40 & 128 & 512 & 512 & 64 & 1.024 & 128 & 1.024 & 32 \\
\hline $4 p$ & $\mathrm{G}$ & 1.024 & G & 128 & 512 & $\mathrm{G}$ & 512 & 64 \\
\hline $4 q$ & 128 & G & G & G & G & G & G & G \\
\hline Flu & 2 & 16 & 2 & $>64$ & 32 & 32 & 64 & 64 \\
\hline
\end{tabular}

a Collection of URM cultures; ${ }^{b}$ American type culture collection. G: growth at the highest concentration tested; Flu: fluconazole. 
(compounds $\mathbf{4 f}, \mathbf{4 g}, \mathbf{4 k}$, respectively), it was not possible to associate substituent position with an increase in activity. For the indole methylidene derivatives $(\mathbf{4} \mathbf{p}$ and $\mathbf{4 q})$, the presence of $\mathrm{NO}_{2}$ in $\mathbf{4} \mathbf{p}$ was essential for antifungal activity. Replacing $\mathrm{NO}_{2}$ with an electron donor group $\left(\mathrm{OCH}_{3}\right.$ in $\left.\mathbf{4 q}\right)$ resulted in a compound without activity.

The importance of the $\mathrm{NO}_{2}$ substituent for antifungal activity in Schiff bases bearing cycloalkyl $[b]$ thiophene derivatives has been discussed by our group. In work reported by Mendonça-Junior et al. ${ }^{26}$ the compound 2-(4-nitrobenzylidene)-amino-4,5,6,7-tetrahydro$4 H$-benzo[ $b]$ thiophene-3-carbonitrile $(6 \mathrm{CN} 10)$ was found to be the most active against Candida and C. neoformans strains.

In general, the presence of electron donor groups, observed in 4a $\left(4-\mathrm{OCH}_{3}\right), 4 \mathbf{h}(4-\mathrm{OH}), 4 \mathbf{i}\left(4-\mathrm{CH}_{3}\right)$, $\mathbf{4 j}\left(4-\mathrm{N}\left(\mathrm{CH}_{3}\right)_{2}\right.$ and $\mathbf{4 q}\left(5-\mathrm{OCH}_{3}\right)$, leads to poor antifungal activity. The only exception observed was compound $\mathbf{4 l}$, which presents three methoxyl substituents $\left(\mathrm{OCH}_{3}\right)$ at positions 3,4 and 5 , being equipotent to fluconazole against T. rubrum 6753 ( $\mathrm{MIC}=64 \mu \mathrm{g} \mathrm{mL}-1$ ), and against T. tonsurans $700\left(\mathrm{MIC}=128 \mu \mathrm{g} \mathrm{mL}^{-1}\right.$ ), where resistance to fluconazole is defined at an MIC $\geq 64 \mu \mathrm{g} \mathrm{mL}^{-1}$.

Similar results were observed in the study conducted by Wang et al. ${ }^{67}$ where it was found that the presence of an electron-donating group such as $4-\mathrm{OCH}_{3}$, or $4-\mathrm{CH}_{3}$ was harmful to the antifungal activity of fenfuram-diarylamine hybrids against phytopathogenic fungi.

Both type and position of halogens at the benzylidene moiety present significant effects on antifungal activity. For the mono-substituted derivatives $\mathbf{4 c}(4-\mathrm{F}), \mathbf{4 b}(4-\mathrm{Cl})$, and 4o (4-Br), the size of the halogen was a great influence on both activity and spectrum of action.

Compound 4o, containing the bromine atom (with the highest atomic volume), was the only derivative causing sensitivity in all tested fungal isolates, and presenting better activity than fluconazole, when compared against the $T$. tonsurans $700\left(\mathrm{MIC}=64 \mu \mathrm{g} \mathrm{mL}^{-1}\right)$ and $T$. rubrum $6753\left(\mathrm{MIC}=32 \mu \mathrm{g} \mathrm{mL}^{-1}\right)$ isolates.

For the di-substituted halogenated derivatives $\mathbf{4 d}$ $(2,4-\mathrm{Cl}), \mathbf{4} \mathbf{e}(2,6-\mathrm{Cl})$, and $\mathbf{4 n}(2,3-\mathrm{Cl})$, the presence of the two chlorine atoms in the ortho position $(2,6-\mathrm{Cl})$ increased the spectrum of activity for compound $\mathbf{4 e}$. Compounds $\mathbf{4 d}$ and 4n were only active against E. floccosum 6999, with respective MICs $=128$ and $256 \mu \mathrm{gL}^{-1}$. Compound $4 \mathrm{e}$ was active against $T$. tonsurans $2822\left(\mathrm{MIC}=256 \mu \mathrm{g} \mathrm{mL}^{-1}\right)$, T. tonsurans $700\left(\mathrm{MIC}=64 \mu \mathrm{g} \mathrm{mL}^{-1}\right)$, and T. rubrum 6753 $\left(\mathrm{MIC}=32 \mu \mathrm{g} \mathrm{mL}^{-1}\right.$ ). Its antifungal activity against these last two isolates was superior to fluconazole.

The importance of the presence of halogens for the antifungal activity of new drug candidates is widely described. In a study conducted by Sui et al., ${ }^{23}$ the most active imine derivatives bearing a kakuol moiety against four phytopathogenic fungi were those presenting halogens, especially chlorine in position 2 (ortho). The same authors also demonstrated that the presence of two chlorine atoms is beneficial for both antifungal activity, and antibacterial activity against Gram-positive bacteria. Karthikeyan et al. ${ }^{21}$ also reports that Schiff bases containing 2,4-dichloro5-fluorophenyl substituents present antifungal activity equal to fluconazole against strains of Aspergillus flavus, A. fumigatus, Penicillium marneffei, and T. mentagrophytes. Echevarria et al..$^{24}$ reported that Schiff bases bearing a piperonyl moiety containing a halogen $(\mathrm{Cl}, \mathrm{Br}$, or I) in position 4 (para) were the most active compounds against E. floccosum and T. rubrum.

According to Mendonça-Junior et al. ${ }^{26}$ the presence of halogens (chlorine and fluorine) is closely related to the chemical structures found in most azole derivatives, such as miconazole, clotrimazole, econazole, and ketoconazole. Halogens contribute to increase lipophilicity for the compounds, facilitating their membrane permeability, and allowing more intense hydrophobic interactions with specific enzymes and receptors. ${ }^{21}$

\section{Conclusions}

A series of novel Schiff bases bearing 2-aminothiophene derivatives was designed, synthesized and spectroscopically characterized. The use of microwave-assisted synthesis produced the compounds in shorter reaction times, with good yields, and using an inorganic base; thus, being an eco-friendly procedure based on principles of green chemistry. The importance of the cycloalkyl ring attached to the 2-aminothiophene core for antifungal activity was investigated using molecular simplification. The cycloakyl ring of cycloalka[b]thiophenes was therefore excluded, giving rise to $\mathrm{C}-4$ and $\mathrm{C}-5$ unsubstituted 2-aminothiophenes. The reduction of "molecular obesity" (lower MW, and $\log \mathrm{P}$ ) promoted by the removal of the cycloalkyl ring resulted in compounds with better druglikeness, adequate pharmacokinetic (ADMET) profiles, low toxicity, and good antifungal profiles, in some cases better than the reference drug.

The SAR revealed that the halogen $(\mathrm{F}, \mathrm{Cl}$, and $\mathrm{Br})$ and nitro $\left(\mathrm{NO}_{2}\right)$ substituents in the benzylidene moiety led to the most active compounds.

Taken together, these results demonstrate that the molecular simplification strategy was a success, improving druglikeness, pharmacokinetic profiles, and water solubility; while increasing antifungal activity for Schiff bases bearing 2-aminothiophene derivatives, thus making them promising lead compounds for the development of new antifungal drugs. 


\section{Supplementary Information}

Supplementary information (pharmacokinetics (ADMET) parameters of all compounds, cytotoxicity graphs of the most active compounds, NMR and MS spectra of all new compounds) is available free of charge at http://jbcs.sbq.org.br as PDF file.

\section{Acknowledgments}

We would like to extend our gratitude to our supporters and partners: Federal University of Pernambuco (UFPE), and Paraíba State University (UEPB). The authors would like to thank Prof Thiago M. de Aquino of the Federal University of Alagoas (UFAL), the Nucleus of Analysis and Research in Nuclear Magnetic Resonance of Alagoas for all NMR spectra acquisitions, and the LCMA-Analytical Center of the Federal University of Paraíba (UFPB) for obtaining all HRMS. The authors thank the Coordenação de Aperfeiçoamento de Pessoal de Nível Superior (CAPES) and the Conselho Nacional de Desenvolvimento Científico e Tecnológico (CNPq) for the scholarships of W. W. Neves and I. S. Luna. This work was supported by CNPq grant number 308590/2017-1. This study was financed in part by the Coordenação de Aperfeiçoamento de Pessoal de Nível Superior Brazil (CAPES), finance code 001.

\section{Author Contributions}

Isadora S. Luna was responsible for data curation, formal analysis, investigation, writing original draft; Wendell W. Neves for data curation, formal analysis, investigation, writing original draft; Reginaldo G. de LimaNeto for data curation, resources, writing review; Amanda P. B. Albuquerque for formal analysis, investigation, writing original draft; Maíra G. R. Pitta, for funding acquisition, resources, validation, review; Moacyr J. B. de Melo Rêgo for funding acquisition, resources, validation, review; Rejane P. Neves for funding acquisition, resources, validation, review; Marcus T. Scotti for conceptualization, resources, software, validation, review and editing; Francisco J. B. Mendonça-Junior, for conceptualization, funding acquisition, resources, project administration, validation, review and editing.

\section{References}

1. Brown, D. G.; Denning, D. W.; Gow, N. A.; Levitz, S. M.; Netea, M. G.; White, T. C.; Sci. Transl. Med. 2012, 4, 165.

2. de Oliveira, D. B. C.; Silva, L. B.; da Silva, B. V.; Borges, T. C.; Marques, B. C.; dos Santos, M. B.; de Oliveira, L. F.; Bolzani,
V. S.; Rodrigues, A. R. A.; Regasini, L. O.; Andrade, A. A.; J. Appl. Microbiol. 2019, 127, 1362.

3. Khurana, A.; Sardana, K.; Chowdhary, A.; Fungal Genet. Biol. 2019, 103255.

4. Singh, A.; Masih, A.; Khurana, A.; Singh, P. K.; Gupta, M.; Hagen, F.; Meis, J. F.; Chowdhary, A.; Mycoses 2018, 61, 477.

5. Bishnoi, A.; Vinay, K.; Dogra, S.; Lancet Infect. Dis. 2018, 18, 250.

6. Pai, V.; Ganavalli, A.; Kikkeri, N. N.; Indian J. Dermatol. Venereol. 2018, 63, 361.

7. Tuon, F. F.; Florencio, K. L.; Rocha, J. L.; Med. Mycol. 2018, $57,265$.

8. Allen, D.; Wilson, D.; Drew, R.; Perfect, J.; Expert Rev. AntiInfect. Ther. 2015, 13, 787.

9. Vermes, A.; Guchelaar, H. J.; Dankert, J.; J. Antimicrob. Chemother. 2000, 46, 171.

10. Stover, K. R.; Farley, J. M.; Kyle, P. B.; Cleary, J. D.; Expert Opin. Drug Saf. 2014, 13, 5.

11. Keller, K. A.; J. Exot. Pet Med. 2012, 21, 181.

12. Pfaller, M. A.; Am. J. Med. 2012, 125, S3.

13. Dharmaiah, S.; Sherif, R. A.; Mukherjee, P. K. In Antifungal Drug Resistance: Significance and Mechanisms; Ghannoum, M. A.; Perfect, J. R., eds.; CRC Press: New York, USA, 2019, ch. 4.

14. da Silva, C. M.; da Silva, D. L.; Modolo, L. V.; Alves, R. B.; de Resende, M. A.; Martins, C. V. B.; de Fátima, Â.; J. Adv. Res. 2011, 2,1 .

15. Wardakhan, W. W.; Gaber, H. M.; Ouf, S. A.; Sherif, S. M.; Phosphorus, Sulfur Silicon Relat. Elem. 2005, 180, 601.

16. Bozorov, K.; Nie, L. F.; Zhao, J.; Aisa, H. A.; Eur. J. Med. Chem. 2017, 140, 465.

17. Huang, Y.; Dömling, A.; Mol. Diversity 2011, 15, 3.

18. Wen, L.; Jian, W.; Shang, J.; He, D.; Pest Manage. Sci. 2019, 75,1123 .

19. Luca, L. D.; Curr. Med. Chem. 2006, 13, 1.

20. Sui, G. Q.; Zhang, W.; Zhou, K.; Li, Y.; Zhang, B.; Xu, D.; Zou, Y.; Zhou, W.; Chem. Pharm. Bull. 2017, 65, 82.

21. Karthikeyan, M. S.; Prasad, D. J.; Poojary, B.; Bhat, K. S.; Holla, B. S.; Kumari, N. S.; Bioorg. Med. Chem. 2006, 14, 7482.

22. Hejchman, E.; Kruszewska, H.; Maciejewska, D.; SowirkaTaciak, B.; Tomczyk, M.; Sztokfisz-Ignasiak, A.; Jankowski, J.; Młynarczuk-Biały, I.; Monatsh. Chem. 2019, 150, 255.

23. Sui, G.; Xu, D.; Luo, T.; Guo, H.; Sheng, G.; Yin, D.; Ren, L.; Hao, H.; Zhou, W.; Bioorg. Med. Chem. Lett. 2020, 30, 126774.

24. Echevarria, A.; Nascimento, M. G.; Gerônimo, V.; Miller, J.; Giesbrecht, A.; J. Braz. Chem. Soc. 1999, 10, 60.

25. Panneerselvam, P.; Nair, R. R.; Vijayalakshmi, G.; Subramanian, E. H.; Sridhar, S. K.; Eur. J. Med. Chem. 2005, 40, 225.

26. Mendonça-Junior, F. J. B.; Lima-Neto, R. G.; de Oliveira, T. B.; de Lima, M. C. A.; Pitta, I. R.; Galdino, S. L.; da Cruz, R. M. D.; de Araújo, R. S. A.; Neves, R. P.; Lat. Am. J. Pharm. 2011, 30, 1492. 
27. Scotti, L.; Scotti, M. T.; Lima, E. O.; da Silva, M. S.; de Lima, M. C. A.; Pitta, I. R.; de Moura, R. O.; Oliveira, J. G. B.; Cruz, R. M. D.; Júnior, F. J. B. M.; Molecules 2012, 17, 2298.

28. Souza, B. C. C.; de Oliveira, T. B.; Aquino, T. M.; de Lima, M. C.; Pitta, I. R.; Galdino, S. L.; Lima, E. O.; Gonçalves-Silva, T.; Militão, G. C.; Scotti, L.; Scotti, M. T.; Mendonça-Junior, F. J. B.; Acta Pharm. 2012, 62, 221.

29. Eleamen, G. R. A.; da Costa, S. C.; Lima-Neto, R. G.; Neves, R. P.; Rolim, L. A.; Rolim-Neto, P. J.; Moura, R. O.; de Aquino, T. M.; Bento, E. S.; Scotti, M. T.; Mendonça-Junior, F. J. B.; Mendonça, E. A. M.; Oliveira, E. E.; J. Braz. Chem. Soc. 2017, 28, 116.

30. Guimarães, G. P.; Reis, M. Y. F. A.; da Silva, D. T.; Junior, F. J.; Converti, A.; Pessoa Jr., A.; Damasceno, B. P. L.; da Silva, J. A.; Braz. J. Microbiol. 2014, 45, 545.

31. Neves, W. W.; Neves, R. P.; Macêdo, D. P. C.; Eleamen, G. R. A.; Kretzschmar, E. A. M.; Oliveira, E. E.; Mendonça-Junior, F. J. B.; de Lima-Neto, R. G.; Braz. J. Microbiol. 2020, 51, 647.

32. Wang, S.; Dong, G.; Sheng, C.; Acta Pharm. Sin. B 2019, 9 , 880.

33. Barreiro, E. J.; Quim. Nova 2002, 25, 1172.

34. Crisóstomo, F. R. P.; Carrillo, R.; León, L. G.; Martín, T.; Padrón, J. M.; Martín, V. S.; J. Org. Chem. 2006, 71, 2339.

35. Puterová, Z.; Krutosíkivá, A.; Végh, D.; Arkivoc 2010, 1, 209.

36. Luna, I. S.; da Cruz, R. M. D.; da Cruz, R. M. D.; de Araújo, R. S. A.; Mendonça-Junior, F. J. B.; Curr. Org. Synth. 2018, 15, 1026.

37. Pires, D. E. V.; Blundell, T. L.; Ascher, D. B.; J. Med. Chem. 2015, 58, 4066.

38. Lipinski, C. A.; Lombardo, F.; Dominy, B. W.; Feeney, P. J.; Adv. Drug Delivery Rev. 2001, 46, 3.

39. Han, Y.; Zhang, J.; Hu, C.; Food Chem. Toxicol. 2018, 116, 264.

40. Clinical and Laboratory Standards Institute (CLSI); M38-A2 Reference Method for Broth Dilution Antifungal Susceptibility Testing of Filamentous Fungi, Approved Standard, $2^{\text {nd }}$ ed.; CLSI: Wayne, PA, USA, 2008, available at: https://clsi.org/ media/1455/m38a2_sample.pdf, accessed in November 2020.

41. Excel, version Office365; Microsoft Corporation, USA, 2020.

42. Hesse, S.; Perspicace, E.; Kirsch, G.; Tetrahedron Lett. 2007, $48,5261$.

43. Grewal, A. S.; Kumar, K.; Redhu, S.; Bhardwaj, S.; Int. Res. J. Pharm. Appl. Sci. 2013, 3, 278.

44. Lipinski, C. A.; Drug Discovery Today: Technol. 2004, 1, 337.

45. Veber, D. F.; Johnson, S. R.; Cheng, H. Y.; Smith, B. R.; Ward, K. W.; Kopple, K. D.; J. Med. Chem. 2002, 45, 2615.

46. Merlot, C.; Drug Discovery Today 2010, 15, 16.

47. Algahtani, S.; Expert Opin. Drug Metab. Toxicol. 2017, 13, 1147.
48. Ghost, J.; Lawless, M. S.; Walman, M.; Gombar, V.; Fraczkiewicz, R. In Silico Methods for Predicting Drug Toxicity, vol. 1425; Benfenati, E., ed.; Humana Press: New York, 2016, ch. 4 .

49. Nisha, C. M.; Kumar, A.; Vimal, A.; Bai, B. M.; Pal, D.; Kumar, A.; J. Mol. Graphics Modell. 2016, 65, 100.

50. Horie, K.; Tang, F.; Borchardt, R. T.; Pharm. Res. 2003, 20, 161.

51. Ferreira, S. B.; Dantas, T. B.; Silva, D. F.; Ferreira, P. B.; Melo, T. R.; Lima, E. O.; Curr. Top. Med. Chem. 2018, 18, 2186.

52. Hodgson, J.; Nat. Biotechnol. 2001, 19, 722.

53. Honório, K. M.; Moda, T. L.; Andricopulo, A. D.; Med. Chem. 2013, 9, 163.

54. Li, A. P.; Drug Discovery Today 2001, 6, 357.

55. Ekowati, J.; Diyah, N. W.; Nofianti, K. A.; Hamid, I. S.; Siswandono, S.; ITB J. Math. Fundam. Sci. 2018, 50, 203.

56. Shehzadi, N.; Hussain, K.; Islam, M.; Bukhari, N.; Khan, M. T.; Salman, M.; Siddiqui, N. I. A.; Rherman, U.; Abbasi, M. A.; Lat. Am. J. Pharm. 2016, 35, 1991.

57. Boguslavsky, J.; Drug Discovery Dev. 2001, 4, 26.

58. Xu, C.; Cheng, F.; Cheng, L.; Du, Z.; Li, W.; Liu, G.; Lee, P. W.; Tang, Y.; J. Chem. Inf. Model. 2012, 52, 2840.

59. Sanguinetti, M. C.; Jiang, C.; Curran, M. E.; Keating, M. T.; Cell 1995, 81, 299.

60. Wang, S.; Li, Y.; Xu, L.; Li, D.; Hou, T.; Curr. Top. Med. Chem. 2013, 13, 317.

61. Zhou, Z.; Gong, Q.; Ye, B.; Fan, Z.; Makielski, J. C.; Robertson, G. A.; January, C. T.; Biophys. J. 1998, 74, 230.

62. Bissell, D. M.; Gores, G. J.; Laskin, D. L.; Hoofnagle, J. H.; Hepatology 2001, 33, 1009.

63. Gomes-Lechon, M. J.; Lahoz, A.; Gombau, L.; Castell, J. V.; Donato, M. T.; Curr. Pharm. Des. 2010, 16, 1963.

64. Chen, M.; Suzuki, A.; Borlak, J.; Andrade, R. J.; Lucena, M. I.; J. Hepatol. 2015, 63, 503.

65. Lima-Neto, R. G.; Cavalcante, N. N. M.; Srivastava, R. M.; Mendonça Jr., F. J. B.; Wanderley, A. G.; Neves, R. P.; dos Anjos, J. V.; Molecules 2012, 17, 5882.

66. Zimmermann, L.; Bussiere, A.; Ouberai, M.; Baussanne, I.; Jolivalt, C.; Mingeot-Leclercq, M.-P.; Decout, J.-L.; J. Med. Chem. 2013, 56, 7691.

67. Wang, H.; Gao, X.; Zhang, X.; Jin, H.; Tao, K.; Hou, T.; Bioorg. Med. Chem. Lett. 2017, 27, 90.

Submitted: September 26, 2020 Published online: January 7, 2021 